\section{Reststoffen den Rest geben}

\begin{abstract}
In jedem Betrieb besteht der stoffliche und energetische Output zu einem erheblichen Teil aus Abfall, Abwasser, Abluft und Abwärme. Diese Reststoffe verursachen vielfältige Kosten und wenig Erlöse. Die Reststoffkostenrechnung hilft, diese Kosten zu verringern und trägt so zugleich zum Umweltschułz bei.
\end{abstract}

$\mathrm{D}$

Von Hartmut Fischer as aus den betriebswirtschaftlichen Lehrbüchern vertraute und das heutige betriebliche Denken nach wie vor prägende Bild der betrieblichen Leistungserstellung (siehe Abbildung 1) ist, dass Inputs im Leistungsprozess zu den vom Kunden erwünschten und bezahlten Produkten umgewandelt werden.

Dieses Bild spiegelt sich im Aufbau der Kostenrechnung wider. Die Kostenartenrechnung ist die kostenrechnerische Erfassung der Inputs. Die Kostenstellenrechnung ordnet Kosten auf Prozesse der Leistungserstellung zu. Die Kostenträgerrechnung ordnet Kosten auf die erstellten Produkte zu. Produkte sind in diesem Bild der einzige Output eines Betriebes.

Die betriebliche Praxis zeichnet jedoch ein anderes, in Abbildung 2 wiedergegebenes Bild. In jedem Betrieb besteht der stoffliche und energetische Output zu einem erheblichen Teil aus Abfall, Abwasser, Abluft und Abwärme. Gemeinsam ist diesen Outputs, dass sie nicht in das vom Kunden erwünschte und bezahlte Produkt eingehen. Diese Outputs werden im Weiteren unter dem Begriff Reststoffe zusammengefasst. Verwandte Begriffe sind Kuppelprodukte, Abprodukte, Rückstände, Non-Product Output, Loss.

\section{Was sind Reststoffkosten?}

Reststoffe verursachen Betrieben vielfältige Kosten und - in sehr begrenztem Umfang - auch Erlöse. Sie werden im Einkauf als Roh-, Hilfs- oder Betriebsstoffe bezahlt, von der Materialwirtschaft gelagert und transportiert sowie vor Anfall in der Produktion oft noch bearbeitet. Diese Kosten werden im Weiteren als die Kosten der Herstellung von Reststoffen zusammengefasst.

Nachdem sie erkennbar als Abfall, Abwasser oder Abluft angefallen sind, werden Reststoffe innerbetrieblich erfasst, teilweise noch behandelt und aufbereitet (zum Beispiel durch Abwasserbehandlung) und dann entweder im Betrieb wieder eingesetzt oder an Dritte abgegeben. Diese Kosten

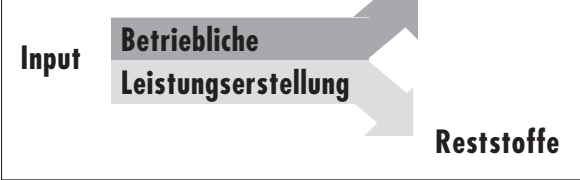

Quelle: eigene Darstellung

werden im Weiteren als die Kosten der Entsorgung von Reststoffen zusammengefasst. Verwertungserlöse werden als negative Kosten der Reststoffentsorgung behandelt.

Die Gesamtheit dieser Kosten und Erlöse wird als Reststoffkosten zusammengefasst. Hierbei wird ein Vollkostenansatz zugrunde gelegt, wenn dies mit dem bestehenden Kostenrechnungssystem vereinbar ist.

Reststoffkosten sind Ausdruck nicht wertschöpfenden Aufwands. Der Kunde honoriert diesen Aufwand in keiner Weise, da er das Produkt nicht wertvoller macht. Der Betrieb dagegen zahlt für jedes Kilogramm Reststoff doppelt: einmal bei dessen Herstellung und dann noch einmal bei der Entsorgung.

\section{Typische Steuerungsdefizite}

In der Industrie sind folgende Defizite bei der Steuerung von Reststoffkosten anzutreffen, die zur Folge haben, dass zahlreiche Öko-Effizienz-Potenziale nicht erschlossen werden.

- Kenntnis der vollen Höhe der Reststoffkosten: Zum einen kennt derzeit kaum ein Unternehmen die volle Höhe seiner Reststoffkosten. Das Rechnungswesen weist nur einen Bruchteil der Reststoffkosten als von Reststoffen verursacht aus. Es ist zwar üblich, einen Teil des Entsorgungsaufwands in gesonderten Kostenarten und

Kostenstellen zu erfassen. Die vollen Kosten der Reststoffe betragen in der Regel jedoch das Zweibis Zehnfache dieser Werte. Die Geschäftsleitung unterschätzt deshalb regelmäßig die wirtschaftliche Bedeutung einer verbesserten Öko-Effizienz und baut dem entsprechend auch nur reduzierten Handlungsdruck auf.

- Kostenzuordnung und Verantwortung: Erfahrungsgemäß sind Produktdesign, Einsatzmaterialien und Gestaltung der Fertigungsprozesse bestimmende Faktoren für die Höhe der Reststoffkosten. Die dafür verantwortlichen Entscheidungsträger und Prozesse werden jedoch nur zum Teil verursachungsgerecht belastet: In Gemeinkosten enthaltene Reststoffkosten werden zum Beispiel oft pauschal zugeordnet. Die Kostenrechnung gibt damit auch Entscheidungsträgern unterhalb der Geschäftsleitung nur begrenzt Anreize und Hilfestellung für die Senkung der Reststoffkosten.

\section{Vorgehen der Reststoffkosten- rechnung}

Zur Überwindung dieser Defizite wurde die Reststoffkostenrechnung entwickelt, deren Grundlagen 1992 gelegt wurden und die der Verfasser seitdem in mehreren Schritten bis 2001 weiterentwickelt hat (1). Kernelemente der Reststoffkostenrechnung sind:

- Erfassung der Reststoffkosten,

verursachungsgerechte Zuordnung der Reststoffkosten zu Reststoffen, Kostenstellen, Kostenträgern und Einsatzmaterialien,

- flexible Auswertung der Reststoffkosten.

Um den Aufwand zu minimieren und die Datenkonsistenz zu maximieren, wird die Reststoffkostenrechnung konsequent in die bestehende Kostenrechnung integriert. Lediglich wo die bestehende Kostenrechnung keine ausreichend gleichartige Funktion bietet, werden Kernelemente der Reststoffkostenrechnung als Nebenrechnung durchgeführt.

Im Folgenden wird ein Verfahren zur Realisierung dieser Reststoffkostenrechnung beschrieben. Das Verfahren sollte bei der Anwendung auf die Besonderheiten des jeweiligen Betriebes angepasst werden. Reststoffkosten-Strukturen, Möglichkeiten der bestehenden Informationssysteme und viele andere Faktoren können Modifikationen im Interesse eines guten Aufwand-NutzenEffektes erforderlich machen. In jedem Fall sind einfache, mit minimalem Zusatzaufwand realisierbare Lösungen anzustreben. 
Im Rahmen der Kostenerfassung und der internen Leistungsverrechnung werden einzelne Kostenbelege (zum Beispiel Materialentnahmebelege, Rechnungen von Zulieferern, Belege für innerbetriebliche Leistungen) durch Kontierung einem oder mehreren Kostensammlern (wie Kostenarten, Kostenstellen, Kostenträgern, Aufträgen oder Projekten) zugeordnet. Für die Reststoffkostenrechnung werden solche Einzelbelege, die reststoffverursachte Kosten abbilden, zusätzlich mit dem Reststoffstrom kontiert, der sie verursacht hat. Zur Kontierung auf Reststoffe kann entweder ein bestehender, nicht anderweitig belegter Kostensammler (zum Beispiel interner Auftrag, Prozess, Projekt) genutzt oder ein zusätzlicher Kostensammler eingerichtet werden.

Diese Kontierung kann und sollte anhand ohnehin erfasster Merkmale der Einzelbelege und dazu hinterlegter Stammdaten automatisiert erfolgen. Dies minimiert den Zusatzaufwand für die Reststoffkostenrechnung und sichert durch das weitgehende Ausschalten personenabhängiger Zuordnungen die bereichs- und periodenübergreifende Vergleichbarkeit von Ergebnissen.

Die Reststoffkostenrechnung kann durch entsprechendes Customizing und punktuelle Funktionsergänzung betrieblicher EDV-Systeme mit begrenztem Aufwand in allen großen betrieblichen Informationssystemen (SAP R/3 und anderen) realisiert werden (2).

\section{- Breiter Anwendungsbereich}

Die Reststoffkostenrechnung hat ein breites Anwendungsgebiet, da Reststoffkosten die entscheidungsrelevanten Kosten mehrerer betrieblicher Aufgabenbereiche und Teilbereiche beinhalten. Neben dem betrieblichen Umweltmanagement handelt es sich dabei um Verpackungsmanagement, Ausbeuteoptimierung für Einsatzmaterialien, Teilbereiche des Qualitätsmanagements (Ausschuss, Nacharbeit), Energiemanagement sowie andere Ver- und Entsorgungsleistungen. Diesen Bereichen ist der zentrale Treiber Material- und Energieeffizienz gemeinsam. Sie lassen sich deshalb mit der Reststoffkostenrechnung unter einer einheitlichen kostenrechnerischen Systematik steuern.

\section{- Reststoff- vs. Umweltschutzkosten}

Die Reststoffkostenrechnung unterstützt das Umweltmanagement wirksamer als die heute verbreitete Berechnung der Umweltschutzkosten:
Umweltkostenrechnung beschränkt sich in der Praxis vor allem auf die Erfassung und Zuordnung von Kosten für die Entsorgung von Reststoffen. Die weitaus höheren Kosten für die Herstellung von Reststoffen werden nicht berïcksichtigt. Dadurch wird der kostensenkende Wert des integrierten Umweltschutzes deutlich unterschätzt und umweltbelastenden Suboptimierungen Vorschub geleistet.

- Die Begriffe Umweltkosten oder Umweltschutzkosten legen nahe, dass Umweltschutz zwangsläufig mit Kostenbelastungen verbunden und der zentrale, kostensenkende Ansatz ein reduzierter Umweltschutz ist. Der Begriff Reststoffkosten vermittelt dagegen, dass Umweltschutz- und Kostenziele synergetisch sein können und der zentrale Ansatz die Reststoff-Vermeidung ist. Dies erhöht die Motivation aller Betriebsbereiche, zu den Zielen des Umweltschutzes beizutragen.

\section{Bezüge zur Prozesskostenrechnung}

Die Reststoffkostenrechnung ist der Prozesskostenrechnung eng verwandt. Die Herstellung und Entsorgung von Reststoffen entspricht einem Hauptprozess. Die Reststoffmengen stellen Kostentreiber dar, denen aus allen Kostenstellen, in denen sie Kosten nachvollziehbar verursachen, Kosten zugeordnet werden. Zugrundegelegt wird ein Vollkostenansatz. Aufgrund der weniger stringenten Systematik ist die Reststoffkostenrechnung jedoch wesentlich einfacher zu handhaben.

\section{Anmerkungen}

(1) Vgl. zur Entwicklung z.B. Fischer, H.: Environmental Cost Management vereinigt Umwelttechnik, Produktion und Controlling, in: Blick durch die Wirtschaft vom 25.02.1993, S. 7 oder Fischer, H.: Reststoffkostenrechnung, in: krp-Kostenrechnungspraxis, 1998, Heft 3, S. 140-144. Der aktuelle Stand, inkl. Beschreibung der erprobten Umsetzung in betrieblichen Softwaresystemen ist eingehend dargestellt in: Fischer, H.: Reststoff-Controlling - ein neues Tool zur Steigerung der Material- und Energieeffizienz, Berlin 2001.

(2) Vgl. Fischer, H.: Umweltkostenrechnung, in: Krcmar, H./ Dold, G./ Fischer, H./ Strobel, M./ Seifert, E. (Hrsg.): Informationssysteme für das Umweltmanagement - Das Referenzmodell ECO-Integral, München 2000, S. 245-299.

\section{Der Autor}

Dr. Hartmut Fischer ist Senior Manager bei der Unternehmensberatung Arthur D. Little International. Kontakt: Arthur D. Little International, Kurfürstendamm 237, 10719 Berlin, Fax 0175-13-5806-785, E-mail: fischer.hartmut@adlittle.com

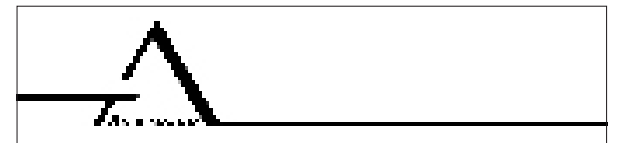

Umweltbewußtsein - Umwelthandeln

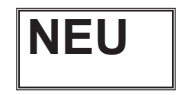

Gundula Hübner und Georg Felser Für Solarenergie. Konsumentenund Umweltpsychologie strategisch anwenden 140 S., kt., DM/SFr.

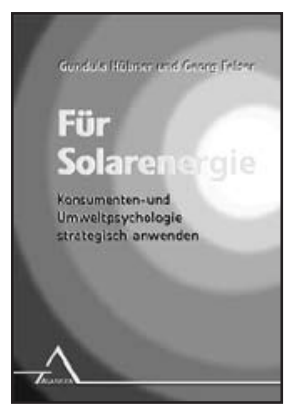
38.-, € 19.-, ISBN 3-89334-341-5

Marcel Hunecke

Ökologische Verantwortung, Lebensstile und Umweltverhalten.

Ausgezeichnet mit dem Förderpreis Umweltpsychologie der Deutschen Gesellschaft für Psychologie

im Jahre 2000.

360 S., kt., DM/SFr. 38.-, € 19.-

ISBN 3-89334-355-5

\section{Sigrun Preuss}

Umweltkatastrophe Mensch.

Über unsere Grenzen und Möglichkeiten, ökologisch bewußt

zu handeln

204 S., kt., DM/SFr. 34.80, € 18.-

ISBN 3-89334-194-3

Sigrun Preuss

Ökopsychosomatik.

Umweltbelastungen und

psycho-vegetative Beschwerden 150 S., kt., DM/SFr. 38.-, € 19.-

ISBN 3-89334-305-9

Volker Preuss (Hrsg.)

Risikoanalysen. Über den Umgang mit Gesundheits- und Umweltgefahren Band 1: 361 S., kt., DM/SFr. 68.-, $€$ 35.ISBN 3-89334-318-0

Band 2: 344 S., kt., DM/SFr. 68.-, € 35.-

ISBN 3-89334-340-7

Gerd Wenninger; Carl Graf Hoyos (Hg.) Arbeits-, Gesundheits- und Umweltschutz. Handwörterbuch verhaltenswissenschaftlicher Grundbegriffe

672 S., geb., DM/SFr. 68.-, € 35.-

ISBN 3-89334-304-0

Asanger Verlag • Heidelberg, Kröning e-mail: verlag@asanger.de Internet: www.asanger.de 
(c) 20I0 Authors; licensee IÖW and oekom verlag. This is an article distributed under the terms of the Creative Commons Attribution Non-Commercial No Derivates License (http://creativecommons.org/licenses/by-nc-nd/3.o/), which permits unrestricted use, distribution, and reproduction in any medium, provided the original work is properly cited. 